\title{
I RECENTI STUDI SULLA BIOLOGIA DI ARISTOTELE COME CONTRIBUTO PER UNA CRITICA ALL'INTERPRETAZIONE DEI NATURPHILOSOPHEN
}

Gaetano Albergo ${ }^{1}$

(Università di Catania)

\section{Introduzione}

Sul finire del XVIII secolo sul suolo tedesco si registra un acceso antagonismo nelle prospettive epistemologiche della scuola kantiana, in parte imputabile alla mediazione operata da Reinhold, il quale con le Briefe über die kantische Philosophie del 1786, e il Versuch einer neuen Theorie des menschlichen Vorstellungsvermögens del 1789 contribuisce a filtrare all'interno del criticismo una forma di teleologismo che avrà presa su quanti gli succederanno a Jena: Fichte, Schelling e Hegel. Dall'equivoco avranno origine $\mathrm{i}$ due fronti dell'interpretazione della gnoseologia kantiana, la Naturphilosophie di matrice idealistica e la Natural philosophy di derivazione britannica. In Germania il concetto di impulso formativo (Bildungstrieb) si diffonde come alternativa sia al meccanicismo new toniano che al riduzionismo evoluzionista. È la biologia l'alternativa al meccanicismo. Il concetto aristotelico di causa finale rivive come concetto cardine di un'epistemologia che in Germania, sulla scorta della reazione all'illuminismo francese e inglese e in linea con l'indirizzo idealistico assunto dalla Critica, attraverso i concetti dell'idealismo trascendentale promuove una nuova filosofia della fisica che riduce lo spazio della ragione al regno dei fini. La coesistenza di Kant e Aristotele è dapprima pura sperimentazione, la causa finale è intesa come forza vitale, poi, con

${ }^{1}$ gaetanoalbergo@yahoo.it

Philosophica, 47, Lisboa, 2016, pp. 27-42. 
Schelling, come anima del mondo e forza organizzatrice dell'organismo. Infine tocca al tipo assumere il ruolo di principio selettivo che semplifica e rende possibile un'organizzazione ordinata degli organismi. Il primato organicista testimonia il processo di snaturamento della Critica. Nella reazione alla metafisica meccanicistica che aveva tanto limitato la fisica, si perde l'equilibrio tra una natura vincolata da leggi necessarie e la creatività della facoltà del giudizio, portando all'emergere di una fisiologia di una natura-naturans spiccatamente teleologica. Nelle morfologie di Goethe, nei suoi concetti di albero e animale originario si profilano le forme epistemologicamente più regressive della Naturphilosophie: la critica romantica al paradigma fisico-meccanicista apre alla restaurazione di una epistemologia autoritaria. Logica e matematica restringono le opzioni delle scienze senza statuto. Di fronte al riemergere di una epistemologia di carattere metafisico-ontologico è possibile però individuare un'alternativa che si candida a portare avanti l'eredità della Critica della ragion pura. Autori come Maimon, Fries, Herbart sviluppano per le scienze emergenti, fra tutte la psicologia e la fisiologia, i presupposti di una teoria della conoscenza che possa legittimarle adottando criteri meno rigidi di quelli dell'epistemologia dominante. La riflessione si attesta su quale metodologia possa garantire una reale crescita conoscitiva. Una volta messo in dubbio il procedimento logico-formale, la conseguenza è, come bene individua Fries, superare la tradizionale connotazione logica dell'induzione, di matrice aristotelica e scolastica, verso un'impostazione metodologica, che, proprio negli anni di Goethe e Hegel, incoraggia l'emergere di un pensiero analitico di cui non si può dar conto nei termini della Naturphilosophie. È forse alla Natural Philosophy che occorre guardare per comprendere come la seconda generazione di kantiani individui il carattere sintetico della filosofia scientifica nelle possibilità che si rendono accessibili qualora la ricerca dei criteri di giustificazione metta al centro il ruolo dell'induzione. La nuova filosofia della natura deve partire dal mettere in discussione la deduzione kantiana e quell' a priori a carattere normativo di una teoria dell'esperienza centrata sulle prestazioni costitutive di una mente ordinatrice. Se questa grande novità era stata introdotta da Kant come reazione al vecchio paradigma ontologico-metafisico, si tratta adesso di garantire la pretesa di sinteticità delle nuove scienze. Tale compito, come osservò Herbart, non può essere perseguito a partire dalla rivoluzione copernicana kantiana, perché l'intelletto "non può trovare nelle cose altro che quello che vi mette esso stesso"2. La biologia, in particolare, non può

2 J.Fr. Herbart, Hauptpuncte der Metaphysik, Göttingen: Baier, 1806. 
assestarsi a partire dall'assunto di una mente che fa la natura, ovvero la funzione legislativa dell'intelletto in rapporto all'esperienza.

\section{Il kantismo di Cuvier e i presunti aspetti regressivi della metafisica teleologica dell'Aristotele naturalista}

All'interno del quadro teorico qui presentato si inserisce la figura del grande naturalista francese Georges Cuvier. Un argomento oggi ampiamente dibattuto non è quanto Cuvier abbia potuto influenzare la nuova filosofia della natura tedesca di inizio XIX secolo, su questo c'è sufficiente accordo, ma quale fattore comune, oltre all'opposizione alla romantische Naturphilosophie, può essere rintracciato tra il 'principio delle condizioni di esistenza' di Cuvier e quella corrente, distinta dalla Naturphilosophie, che è stata battezzata teleomeccanicismo. Scienziati come J.F. Blumenbach, C.H. Kielmeyer, A. von Humboldt, J. Müller, $\mathrm{H}$. Lotze sono stati inseriti in questa corrente, anche se pare più corretto parlare di teleomeccanicisti a proposito di quegli zoologi tedeschi che per primi percepirono le difficoltà a spiegare il concetto di organismo solo in termini meccanicistici. Bisognava trovare una via mediana tra spiegazione causale di stampo teleologico e spiegazione meccanicistica. Secondo Timothy Lenoir (1989) questo sarebbe conseguenza naturale della concezione kantiana di una biologia che non può restare imbrigliata nelle rigide maglie del meccanicismo. Pertanto, solo una prospettiva organicistica può riformulare il problema principale che il filosofo della natura si trova ad affrontare anche in epoca pre-darwiniana. Il riferimento a Cuvier risulta subito scontato. A partire dalla sua formazione alla Karlsschule, dove viene influenzato dagli studi di anatomia comparata del Kielmeyer, passando poi al prestigio di Cuvier a Parigi, durante gli anni che vi vedono affluire, dopo Alexander von Humboldt, una lunga serie di naturalisti allievi di Blumenbach e Reil, tutti soggetti al fascino della rivoluzione iniziata dal naturalista francese.

Di recente, John O. Reiss (2009), nella sua ampia analisi del 'principio delle condizioni di esistenza' di Cuvier, ha sottolineato come tale principio "con la sua enfasi di origine kantiana sull'integrità funzionale dell'organismo nel suo ambiente, era piuttosto congeniale al punto di vista dei giovani tedeschi"3. Questa interpretazione, e con questo entriamo nel

J.O. Reiss, Not by Design: Retiring Darwin's Watchmaker, Oakland CA: University of

California Press, 2009, p. 107. 
merito della questione qui affrontata, si inserisce all'interno del recente dibattito volto a determinare la fonte del principio che consente a Cuvier di dire, con parole ormai famose "l' anatomia comparata ha raggiunto un tale livello di perfezione che, dopo aver ispezionato un singolo osso, è spesso possibile determinare la classe, e a volte anche il genere dell'animale a cui appartiene, soprattutto qualora quell'osso appartenga alla testa o alle membra" ". Secondo Reiss, delle due possibili fonti, rispettivamente il teleologismo aristotelico e quello kantiano, l'idea dell'integrità funzionale dell'organismo, insieme alla tesi della subordinazione funzionale delle parti rispetto all'intero, avrebbero avuto origine solo all'interno di un quadro epistemologico che farebbe di Cuvier un naturalista trascendentale. Vale a dire, se è vero che l'identificazione delle condizioni di esistenza con le cause finali fanno di Cuvier un teorico del teleologismo 'esterno', una sorta di sostenitore dell'argomento del Design ad opera di un creatore, è anche vero che il presupposto sarebbe pur sempre la distinzione kantiana tra un ruolo 'regolativo' del principio di esistenza nella spiegazione dell'organismo animale, e un ruolo 'costitutivo'. Adottare il primo, tipica strategia kantiana, consentirebbe di mantenere le distanze da Aristotele e assestare il teleologismo di Cuvier su una metafisica deflazionata, una sorta di finalismo 'interno', senza cadere in un incipiente teleologismo organicistico dai toni prematuramente darwiniani.

Nella tesi sostenuta da Ross, almeno nel ruolo attribuito a Cuvier, una lettura rinforzata dalla mediazione della tesi di Lenoir, è possibile riconoscere alcuni punti di debolezza sul piano della ricostruzione storica. Inoltre, come vedremo nel prossimo paragrafo, il kantismo attribuito a Cuvier non ha ragione di sussistere attraverso un paragone sbagliato, quello con un Aristotele teorico di teleologismo metafisico in buona parte lontano dall'immagine reale che la storia della filosofia della scienza recente ha contribuito a rendere manifesto.

Che Cuvier non fosse così vicino ai teleomeccanicisti tedeschi lo dimostra il fatto che per essi l'organizzazione funzionale degli organismi, e le leggi che presiedono ad esse, tanto necessarie quanto quelle 'metafisiche', non possono essere l'unico fattore della spiegazione dell'organizzazione gerarchica degli animali. L'accento è posto anche sulla discendenza storica, sulle condizioni materiali che in una certa epoca consentono le possibili trasformazioni. Questo spingerà gli zoologi tedeschi, materialisti che adottano i concetti di 'forza vitale', 'principio di vita' ecc., ad accet-

Cuvier 1798, in M.J.S. Rudwick, Georges Cuvier, Fossil Bones, and Geological Catastrophes, Chicago: University of Chicago Press, 1997, p. 36. 
tare l'embriologia comparata nel loro programma, a differenza di Cuvier. Inoltre, restando in tema, l'idea dell'integrità funzionale di matrice kantiana che Reiss attribuisce saldamente a Cuvier, sembra non essere così originale, se come osservato da Leroi (2014), già Darwin, commentando il principio delle condizioni di esistenza, lo rende congruente e anzi funzionale alla teoria della selezione naturale:

Occorre notare in che modo sottile Darwin cambia il significato di Cuvier. Dove Cuvier invoca le condizioni di esistenza di solito sta cercando di spiegare l'adattamento delle parti dell'animale l'una con l'altra; quando lo fa Darwin, lo fa per spiegare l'adattamento delle parti dell'animale con il suo ambiente. La differenza è solo di enfasi. Chiunque cerchi di capire l'organizzazione degli esseri viventi li studia necessariamente come interi in loro stessi e nel loro mondo; i tre grandi studiosi dell'organizzazione animale, Aristotele, Cuvier e Darwin, hanno tutti e tre posto un occhio su entrambe le cose (Leroi, 2014, p. 285).

\section{La biologia aristotelica tra osservazione e spiegazione causale}

L'idea che la moderna anatomia comparata abbia poco a che spartire con Aristotele si basa sul chiaro fraintendimento che attribuisce ad Aristotele aspetti della teleologia 'esterna' alla maniera del Timeo platonico. Prenderemo in esame quella parte della biologia aristotelica che è tanto servita ai teorici della natura del XIX secolo non solo come materiale da catalogo, ma anche come materia di indagine scientifica, anche se non affatto rispondente ai canoni dell'organizzazione sistematica. Negli studi aristotelici degli ultimi vent'anni, James Lennox è lo studioso che più di altri, approfondendo alcune intuizioni di Balme, ha indagato il modo in cui alcuni aspetti della biologia aristotelica, come l'assenza di una classificazione tassonomica completa e la pratica del metodo della divisione, assieme alla relativa organizzazione dell'informazione riportata, proprio quegli elementi che rappresentano, almeno prima facie, i maggiori ostacoli per il progetto volto a includere un trattato come la Historia tra le opere più propriamente teoretiche, in realtà, sarebbero interpretati nel modo corretto solo presupponendo alcuni principi epistemologici degli Analitici, relativi alla forma propria delle proposizioni scientifiche. Così, a partire dalla fondamentale distinzione formulata negli Analitici Secondi tra conoscenza accidentale e conoscenza in assoluto, Lennox si chiede se l'informazione raccolta per via diairetica possa venire incontro alle raccomandazioni metodologiche sull'uso dell'informazione contenute nel libro II, 14 della stessa opera. 
Aristotele apre il capitolo 2 del libro I degli Analitici Secondi affrontando il problema dell'episteme: "crediamo di conoscere ogni cosa in senso assoluto - però non nella maniera sofistica, cioè in maniera accidentale - quando crediamo di conoscere la causa per la quale la cosa è (dal momento che di ogni cosa vi è una causa) e non può capitare che essa sia in altro modo". Subito dopo Aristotele discute degli errori relativi all'universalità della dimostrazione, tra questi vi è il caso in cui si sbaglia, per la mancanza di un termine generale, un nome per un genere che include diverse entità delle quali si predica qualcosa in modo separato, prese ad una ad una, e ciò per l'incapacità a cogliere, tramite un nome, l'appartenenza a quel genere più ampio che, di fatto, rende possibile l'attribuzione. Poco più avanti Aristotele presenta un altro caso. Qui l'errore non deriverebbe dalla mancanza di termini generali:

Per questo, neppure se si dimostri per ciascun tipo di triangolo, con una dimostrazione o unica o diversa, che ciascuno ha due angoli retti - separatamente quello equilatero e quello scaleno e quello isoscele - non si conosce mai che il triangolo ha due angoli retti, se non in modo sofistico, né riguardo al triangolo in universale, neppure se non esiste nessun altro triangolo oltre questi. In effetti, non lo si sa in quanto triangolo, né si sa che gode di questa proprietà ognuno dei triangoli, se non secondo il numero; ma secondo la specie non si sa che ne gode ognuno, anche se non ne esiste nessuno che non si conosca (74a 26-33).

Come noto, la teoria della dimostrazione di Aristotele, a partire dalla conoscenza di definizioni e principi primi necessari, deve rendere possibile una spiegazione causale di tutte le altre verità universali appartenenti a un certo dominio. Tali forme predicative saranno un riferimento 'primo e universale' all'oggetto solo se individueranno determinazioni che appartengono al soggetto 'universalmente' (katholou), 'per sé' (kath'hauto) e 'come tale' (hei auto). Aristotele dunque introduce questo genere di dimostrazione prendendo un esempio tratto dalla geometria. Dare una dimostrazione scientifica del fatto che tutti i triangoli hanno gli angoli interni uguali a due angoli retti significa spiegare perché tale determinazione appartiene al triangolo in quanto tale. Potremmo nominare diverse determinazioni con cui riferirci a qualcosa che soddisfa l'avere la proprietà di possedere angoli interni pari a due retti, basta pensare a tutti gli oggetti o immagini triangolari. Conoscere in assoluto, però, significa conoscere la ragione in virtù della quale tutte queste cose hanno quella determinazione. Pertanto, sapere che ogni sorta di cosa triangolare è in possesso dell'attributo di avere gli angoli interni pari a due retti, e non sapere che 
tutte hanno quell'attributo in quanto triangoli, significa avere conoscenza solo accidentale. Non è il linguaggio estensionale ad aiutarci a progredire nella dimostrazione, non a caso Aristotele poco prima (73b25-74a3) ha introdotto quel preciso linguaggio intensionale che abbiamo citato sopra.

Secondo Lennox è possibile tradurre col seguente sillogismo quella presunta conoscenza del fatto che forme determinate di un oggetto più universale hanno certe determinazioni perché queste apparterrebbero all'oggetto in quanto tale:

$\mathrm{P}$ (somma degli angoli interni pari a due angoli retti) appartiene a ogni triangolo

Essere un triangolo appartiene a ciascuna figura isoscele

$\mathrm{P}$ appartiene a ciascuna figura isoscele

Pertanto, ponendo la triangolarità come termine medio, si giunge alla conclusione che le figure isosceli devono avere, in modo naturale, gli angoli interni pari a due angoli retti. Il ragionamento di Lennox procede distinguendo due diversi livelli d'uso del linguaggio intensionale, precisando anche quale termine medio sarebbe più corretto per una dimostrazione che porti alla conoscenza in assoluto. Lo schema sillogistico presentato è analizzato da Lennox secondo la seguente formulazione (A). Seguirà una seconda spiegazione (B) adottata da Lennox come secondo livello di una spiegazione scientifica:

A. 1. L'attribuzione da spiegare è quella di una determinazione che appartiene al soggetto necessariamente, ma allo stesso modo anche ad altri soggetti, quello che Aristotele, negli Analitici Secondi, descrive come l'attribuzione meno universale, come il fatto che la somma degli angoli interni risulti pari a due retti si dice del triangolo isoscele.

2. L'attribuzione è spiegata mostrando che il soggetto è una varietà di quella specie alla quale il predicato appartiene come cosa prima e come tale: come il fatto che la somma degli angoli interni risulti pari a due retti si dice del triangolo.

3. Dunque, se dovessimo dimostrare in modo sillogistico, il termine medio identificherebbe il genere prossimo al soggetto, rispetto al predicato in questione. 
B. 1. L'attribuzione da spiegare è quella di una determinazione che appartiene al soggetto come cosa prima e in quanto tale: come il fatto che la somma degli angoli interni risulti pari a due retti si dice del triangolo.

2. Questa attribuzione come cosa prima è spiegata identificando come responsabili alcuni aspetti della particolare natura del soggetto.

3. Dunque il termine medio non identifica un genere più grande del quale il soggetto sarebbe una sottospecie, ma un aspetto della specifica natura del soggetto, cioè qualcosa proprio di esso che lo rende quella particolare entità che esso è.

Queste due diverse formulazioni del rapporto tra determinazione predicata e soggetto di cui si predica sarà ripresa da Aristotele al capitolo 9 del libro I, dove dice:

Conosciamo ciascuna cosa non accidentalmente, quando la conosciamo secondo ciò in base a cui il predicato le appartiene, a partire dai principi propri di quella cosa in quanto quella cosa: per esempio, conosciamo l'avere gli angoli uguali a due retti, conoscendo la cosa a cui la proprietà enunciata appartiene per sé, a partire dai principi propri di questa cosa (76a 4-9)

Secondo Lennox questo confermerebbe l'idea che per Aristotele una spiegazione varrebbe come dimostrazione in assoluto solo se la conclusione stabilisce un'attribuzione come cosa prima e in assoluto, ed è ottenuta tramite caratteristiche proprie di quel soggetto. Potremmo aggiungere qualche osservazione, se dicessimo: 'i monotremi depongono le uova in virtù del fatto di essere monotremi', potremmo intendere questa come un'espressione quasi tautologica, nel senso che presuppone la sola conoscenza che quella caratteristica appartiene a tutti gli esemplari dei monotremi. Sarebbe anche un'affermazione fuorviante se con essa volessimo predicare una caratteristica in assoluto, come dimostrato, perché deporre le uova non è caratteristica unica dei monotremi, anzi, essi sono solo l'eccezione tra i mammiferi. Ed è questo, però, che ci consente di identificare i monotremi al livello di generalità giusto per ottenere maggiore conoscenza di essi. Dunque, se con l'espressione 'in virtù di essere monotremi' intendiamo il fatto che deporre le uova è caratteristica tipica dei monotremi, allora non abbiamo nessuna conoscenza nel senso aristotelico, se invece con 
quell'espressione ci riferiamo ad aspetti unici della natura dei monotremi, al livello giusto di generalità, cioè tra i mammiferi, aspetti che rendono necessaria quella caratteristica, allora progrediamo verso una conoscenza causale. Un altro esempio: 'quell'uccello ha un becco in virtù dell'essere un uccello'. Non è chiaro se il soggetto è identificato come un particolare uccello o come uccello in senso generale, e non è chiaro se l'espressione 'in virtù dell' essere un uccello' indichi il sapere, senza ragioni, che il becco è caratteristica unica degli uccelli, o se invece, identificato l'uccello in generale, ci si riferisca alla conoscenza di aspetti della vita e dell'essere degli uccelli che rendono necessario l'avere un becco. Seguendo le schema di Lennox nel primo caso avremmo una conoscenza solo accidentale, e nel secondo in assoluto.

Sono diversi gli interpreti contemporanei di Aristotele che hanno adottato lo schema di Lennox. Ad esempio, Michael Ferejohn, nel suo studio sulla dimostrazione in Aristotele (1990), individua due forme distinte di dimostrazione. La prima di tipo verticale (tipo B, in Lennox), dove la prima premessa contiene termini non convertibili e produce il tipico sillogismo Barbara:

Tutti i B sono A, e

Tutti i C sono B, dunque

(iii)

Tutti i C sono A

Schematicamente, la relazione tra $\mathrm{i}$ tre termini sarà di tipo verticale. A questa, secondo Ferejohn, Aristotele avrebbe aggiunto forme in cui i termini del sillogismo potrebbero essere connessi in modo laterale, dove il possesso da parte di un genere (C) di un attributo per sé (A) si spiegherebbe facendo riferimento a un altro attributo posseduto per sé (B). La relazione tra $\mathrm{A}$ e B sarebbe di implicazione reciproca, cioè di convertibilità. L'esempio che Ferejohn riprende dalla biologia di Aristotele è il seguente:

(i) Tutti (e solo) gli animali sprovvisti di incisivi hanno un terzo stomaco, e

(ii) Tutti (e solo) gli animali dotati di corna sono sprovvisti di incisivi, dunque

(iii) Tutti (e solo) gli animali dotati di corna hanno un terzo stomaco. 
In tal modo, spieghiamo la presenza di un terzo stomaco negli animali dotati di corna tramite una particolare configurazione dei denti. Sarebbe anche possibile l'inverso, spiegare cioè la particolare configurazione dei denti negli animali con corna per mezzo della presenza del terzo stomaco. Ferejohn condivide l'idea di Lennox, secondo la quale Aristotele nella Historia preferisce spiegazioni di tipo laterale, facendo ampio uso di proprietà coestensive e di co-differenze ${ }^{5}$.

Come risulterà chiaro tra breve, quando prenderemo in esame il ruolo delle definizioni nella spiegazione dei capitoli 16-17 del libro II degli Analitici Secondi, nella prospettiva di Lennox le spiegazioni di tipo A, secondo lo schema di sopra, rappresentano un importante contributo in direzione di spiegazioni di tipo B, e questo perché consentirebbero di formulare attribuzioni universali mediante l'individuazione del genere che possiede il predicato per sé. Toccherà alle spiegazioni di tipo $\mathrm{B}$ dar conto di tali attribuzioni. Rispondere alla domanda: perché il predicato appartiene al genere di cose cui appartiene in quanto tale? Sarà dunque necessario individuare quelle ragioni, proprie del genere, che rendono immediatamente necessario che esso abbia quel predicato.

Le distinzioni fin qui formulate e l'idea che vi sia un modo di organizzare le nostre proposizioni vere in modo da procedere da conoscenze fattuali verso conoscenze causali presentano evidenti riferimenti alle raccomandazioni metodologiche presenti nel libro II, 14 degli Analitici Secondi. Qui Aristotele suggerisce come il metodo dicotomico possa contribuire a trovare soluzioni per i problemi scientifici:

Per avere padronanza dei problemi si devono scegliere le partizioni e le divisioni, e sceglierle in questo modo: dando per supposto che il genere è la determinazione comune di tutti i soggetti: per esempio, se i soggetti studiati siano viventi, quali determinazioni appartengono ad ogni vivente; e, una volta assunte queste, di nuovo quali conseguono al primo dei restanti raggruppamenti nella sua totalità: per esempio, se questo è 'uccello', quali conseguono ad ogni uccello; e così sempre prendendo in considerazione i raggruppamenti più vicini (98a 1-7).

\footnotetext{
5 In modo analogo, Richard McKirahan (1992) riprende lo schema di Lennox per distinguere tra il caso in cui un attributo appartiene in modo universale al suo soggetto primo, nei termini di McKirahan avremo una USA proof, e i casi in cui una proprietà sarà predicata del soggetto non in modo diretto ma in virtù dell'appartenenza del soggetto a un genere universale che possiede quella determinazione in quanto tale (il riferimento è alla spiegazione di tipo A con la quale Lennox tratta l'esempio del triangolo). McKirahan chiama quest'ultimo caso 'Argomento dell'applicazione della prova', per indicare che in questi casi si partirebbe proprio dalle conclusioni universali ottenute dalle USA proof per applicarle a casi particolari.
} 
Al libro I, 31 degli Analitici Primi Aristotele giudica insufficiente la diairesis come metodo per giungere alla conoscenza della cause. Tale strumento, applicato a un dominio, presuppone che vi sia un certo insieme di divisioni già date. È sul piano empirico, dunque, che si decide 'la padronanza' dei problemi, cioè cogliere quali differenze appartengono a un soggetto. In seguito, però, occorrerà chiedersi perché, e individuare un termine medio non più esteso della differenza predicata. Che la padronanza dei problemi abbia inizio con l'individuazione delle differenze al livello empirico trova sostegno al libro I del De Partibus, dove Aristotele, con una domanda retorica, si interroga sul modo di procedere delle ricerche naturali: "Deve lo studioso della natura - al modo stesso dei matematici nelle loro esposizioni sull'astronomia - osservare prima i fenomeni relativi agli animali e le parti di ognuno di essi, per poi spiegare il perché e le cause, oppure procedere in qualche altro modo? (I, $1639 \mathrm{~b} 7-10$ )". La risposta è ovvia, infatti al passo 640a 14-15 troviamo: "Sembra che il punto di partenza debba consistere, come anche in precedenza abbiamo detto, nel raccogliere i fenomeni relativi a ciascun genere, e che si debbano poi esporre le loro cause e trattare della generazione". L'idea che i fatti si colgano indagando le apparenze, e che tale è il metodo da perseguire nelle indagini naturali, riceve definitiva conferma proprio dalle famose parole con le quali Aristotele apre il libro II del De Partibus, dove afferma che l'Historia ha già mostrato di quali e quante parti sia costituito ciascun animale, si tratterà adesso di indagarne le cause.

Il principale suggerimento metodologico contenuto nel passo citato del libro II, 14 è di indagare a partire dal genere comune più esteso ( $\mathrm{ad}$ esempio, essere vivente) al quale appartiene l'oggetto della nostra ricerca, chiedersi quali determinazioni appartengono ad esso necessariamente, per poi procedere con il primo dei raggruppamenti restanti, quello ad esso più vicino (ad esempio, uccello). Di questo andremo a raccogliere tutte le determinazioni comuni ad ogni esemplare, procedendo poi in modo reiterato. Le ragioni di tale pratica sono così formulate da Aristotele:

Infatti è chiaro che già avremo la possibilità di dire 'perché' le determinazioni che conseguono appartengono ai raggruppamenti subordinati al genere comune: per esempio, perché appartengono all'uomo o al cavallo. Sia 'vivente' ciò in luogo di cui si pone $\mathrm{A}$, le determinazioni che conseguono ad ogni vivente siano $\mathrm{B}$, certe specie viventi ciò in luogo di cui si pongono C D E. Ora, è chiaro in forza di che cosa B appartiene a D: in forza di A. Similmente è anche per le altre specie; e nel caso dei raggruppamenti subordinati vale sempre il medesimo discorso (98a 8-12). 
Un esempio utilizzato da Lennox può rendere l'argomento ancora più esplicito. Supponiamo di condurre una ricerca sul falco pellegrino, e di chiederci per quale motivo esso possieda un becco adunco. L'esperienza ci dice che il falco è un uccello, e se dovessimo dirlo nei termini delle dicotomie sapremmo che avere il becco è una determinazione dell'uccello, ma dovremmo attivare anche altre differentiae, le quali ci conducono a cogliere il legame tra l'avere un becco adunco e avere artigli ricurvi, una solida struttura alare che consente il volo elevato, l'essere carnivoro. Potremmo così avere la seguente spiegazione:

\section{Avere un becco adunco appartiene ad ogni uccello con artigli ricurvi}

Essere un uccello con artigli ricurvi appartiene a ogni falco pellegrino

Dunque, avere un becco adunco appartiene a ogni falco pellegrino

Questo genere di divisione ci consente anche di capire quanto sia importante non trascurare i generi diversi da quello primo. Emerge, dunque, che l'Historia Animalium sarebbe un trattato organizzato per offrire informazioni adatte a spiegare perché una caratteristica è predicata di un soggetto in modo accidentale, e utili a consentire che la ricerca sia orientata verso spiegazioni di forme di predicazione in universale ${ }^{6}$.

6 Tra i più strenui oppositori della tesi di Lennox qui presentata si trova indubbiamente R. Lloyd. Secondo l'autore inglese sarebbero diversi i motivi per rifiutare la tesi che pone gli studi biologici in linea con le austere coordinate scientifiche degli Analitici Secondi. In particolare, è possibile mantenere l'impianto sillogistico, assiomaticodeduttivo, degli Analitici se l'oggetto della ricerca consiste di organismi vivi, posti in un contesto, composti di materia e forma? Perché non attingere all'eterogeneità del concetto di dimostrazione che è facile rintracciare in altre opere di Aristotele, e a forme diverse di necessità, per meglio indagare quei particolari oggetti della ricerca biologica? È possibile ricondurre il concetto di divisione in atto nella biologia, operante su diverse differentiae simultaneamente, nel solco degli Analitici? Per Lloyd non è in discussione il fatto che nelle opere biologiche ci sia una forma di dimostrazione, il problema è che non è la stessa di quella presentata negli Analitici, neanche a condizione di considerare questa come un puro ideale. Questo vale anche per un'opera più scientificamente impostata come il De Partibus, infatti secondo Lloyd: "Parts of Animals I,1 has, rather, to be taken on its own terms, as allowing a mode of demonstration that does not conform to the criteria of Posterior Analytics, most notably in that it does not take as its starting-points the primary indemonstrables identified in Posterior Analytics I, 2, but uses end-products as its starting-points and works back to their conditionally necessary antecedents. While the reasoning is deductive, the nature of the premises, the mode of necessity and the goal of the inquiry all differ from those in view in the opening chapters of Posterior Analytics I" (G.E.R. Lloyd, Aristotelian Explorations, Cambridge UK: Cambridge University Press, 1996, pp. 36-37). 
In conclusione, abbiamo sottolineato più volte che lo scopo della Historia Animalium è quello di cogliere le differentiae e gli attributi che appartengono a tutti gli animali. Poi si tratterà di indagare le cause. Questa sequenza potrebbe essere anche intesa in termini di valore. Potremmo pensare che si abbia scienza solo quando si persegue la seconda parte della ricerca. Eppure, come abbiamo qui evidenziato, trattando prima dell'ampio spettro di differentiae tra gli animali messe in luce da Aristotele, e poi del background teoretico che gli Analitici offrono per comprendere lo spessore teorico dell'Historia, pare evidente ormai che è lo stesso Aristotele a suggerirci che sia proprio quella la sequenza, la logica che, se seguita in modo appropriato, renderà possibile distinguere tra le cose a partire dalle quali la dimostrazione procede e le cose a proposito delle quali cercheremo dimostrazioni.

\section{Conclusioni}

L'impresa realizzata da Aristotele nelle sue indagini sul mondo naturale, in particolare biologico, ha come sfondo due assunti teorici: la possibilità di organizzare i phainomena in modo dialetticamente ben strutturato ma allo stesso tempo aperto, flessibile, come la realtà vivente che si studia, e l'opportunità di offrire alla conoscenza teoretica, di tipo assiomatico, non solo informazioni e strumenti per la comprensione delle singole specie, ma anche metodi, e le relative logiche, che, se ben seguiti, condurranno alla conoscenza scientifica. Questa, intesa nell'accezione di conoscenza causale, non può procedere in modo puramente formale. L'idea di causa si dice in molti modi. Pertanto, il sillogismo da solo non riesce a individuare il preciso livello di spiegazione causale che il termine medio è chiamato a svolgere. La causazione potrebbe vertere sulla materia comune a un gruppo, sul fatto primo inteso come 1' "in forza di che cosa", oppure sulla funzione, o il fine, cioè l' "in vista di qualcosa". Scegliere solo la via sillogistica significherebbe rendere la causazione implicita, è per questo motivo che abbiamo bisogno di spiegazioni non formali.

Allo stesso tempo, questo non significa rinunciare alla possibilità di far uso di informazione sistematicamente organizzata per strutturare spiegazioni secondo livelli di analisi rispondenti a problemi diversi. La natura del metodo espositivo non può tradire la dottrina aristotelica, che non formula alternativa alla validità universale del metodo che pone in rapporto logico l'osservazione dei phainomena e la dichiarazione del dià tì e delle aitiai. Gli Analitici, sostiene Lennox, non esigono di scrivere ogni buona spiegazione scientifica in termini sillogistici, è sufficiente che quella spiegazione sia, in linea di principio, riformulabile in termini sillogistici. 


\section{BIBLIOGRAFIA}

Opere di Aristotele citate:

Opere biologiche, a cura di D. Lanza e M. Vegetti, Torino: Utet, 1971 (1996).

Organon, a cura di M. Zanatta, 2 voll., Torino: Utet, 1996.

La Metafisica, a cura di G. Reale, 2 voll., Napoli, 1968.

\section{La critica:}

Balme, D.M., (1962), "ГENO $\Sigma$ and EI $\triangle \mathrm{O} \Sigma$ in Aristotle's Biology”, Classical Quarterly, Oxford: OUP, pp. 81-98.

- (1987), Aristotle's Use of Division and Differentiae, in Gotthelf and Lennox (eds.), pp. 69-89.

Bourgey, L., (1955), Observation et Expérience chez Aristote, Paris: Vrin.

Ferejohn, M., (1991), The Origins of Aristotelian Science, New Haven: Yale University Press.

Gotthelf, A., J.G. Lennox, (eds.) (1987), Philosophical Issues in Aristotle's Biology, Cambridge UK: Cambridge University Press.

Herbart, J.Fr., (1806), Hauptpuncte der Metaphysik, Göttingen: Baier.

Lennox, J.G., (2001), Aristotle's Philosophy of Biology, Cambridge UK: Cambridge University Press.

Lenoir, T., (1982), The Strategy of Life: Teleology and Mechanics in Nineteenth Century German Biology, Dordrecht and Boston: D. Reidel.

Leroi, A.M., (2014), The Lagoon. How Aristotle Invented Science, New York: Viking Pinguin Group.

Linneo, C., (1758), Systema Naturae per Regna Tria Naturae, secundum classes, ordines, genera, species, cum characteribus, differentiis, synonymis, locis, Holmiae, Imprensis Laurentii Salvii, 2 volumi.

Lloyd, G.E.R., (1996), Aristotelian Explorations, Cambridge UK: Cambridge University Press.

Luis, P., (1956), "Observations sur le vocabulaire technique d'Aristote", in Mélanges Diès, Paris, pp. 141-149.

Manquat, M., (1932), Aristote Naturaliste, Paris.

McKirahan, R.D., (1992), Principles and Proofs: Aristotle's Theory of Demonstrative Science, Princeton NJ: Princeton University Press. 
Platone, (1984), Opere complete, a cura di G. Giannantoni, Roma-Bari: Laterza. Reinhold, K.L., (1786), Briefe über die kantische Philosophie.

— (1789), Versuch einer neuer Theorie des menschlichen Vorstellungsvermögens. Reiss, J.O., (2009), Not by Design: Retiring Darwin's Watchmaker, Oakland CA, University of California Press.

Riondato, E., (1961), Storia e metafisica nel pensiero di Aristotele, Padova: Editrice Antenore.

Rudwick, M.J.S., (1997), Georges Cuvier, Fossil Bones, and Geological Catastrophes, Chicago: University of Chicago Press.

\section{SOMMARIO}

L'impresa realizzata da Aristotele nelle sue indagini sul mondo naturale, in particolare biologico, ha come sfondo due assunti teorici: la possibilità di organizzare i phainomena in modo dialetticamente ben strutturato ma allo stesso tempo aperto, flessibile, come la realtà vivente che si studia, e l'opportunità di offrire alla conoscenza teoretica, di tipo assiomatico, non solo informazioni e strumenti per la comprensione delle singole specie, ma anche metodi, e le relative logiche, che, se ben seguiti, condurranno alla conoscenza scientifica. Questa, intesa nell'accezione di conoscenza causale, non può procedere in modo puramente formale. Il nostro obiettivo è di dimostrare perché i Naturphilosophen non hanno colto la lezione aristotelica, finendo con il rifiutare il suo teleologismo perché considerato metafisicamente regressivo.

Parole chiavie: La biologia di Aristotele - Il principio delle condizioni di esistenza di Cuvier - Naturphilosophie - Teleologia-Teleomeccanicismo

\footnotetext{
ABSTRACT

The work realized by Aristotle in his investigations on the natural world, in particular the biological world, has as backdrop two theoretical assumptions: the ability to organize phainomena in such a dialectically well structured, although at the same time open and flexible way, as the living reality that is studied, and the opportunity to offer to the theoretical knowledge, of axiomatic nature, not only information and tools for the understanding of individual species, but
} 
also methods, and its logic, which, if properly pursued, will lead to scientific knowledge. This, understood in the sense of causal knowledge, cannot be pursued in a purely formal way. Our aim is to demonstrate why Naturphilosophen did not get the Aristotelian lesson, up to refuse his teleologism because considered metaphysically regressive.

Keywords: Aristotle's Biology - Cuvier's principle of conditions for existence Naturphilosophie - Teleology - Teleomechanism 